\title{
Identification of Levothyroxine Antichagasic Activity through Computer-Aided Drug Repurposing
}

\author{
Carolina L. Bellera, ${ }^{1}$ Darío E. Balcazar, ${ }^{2}$ Lucas Alberca, ${ }^{1}$ Carlos A. Labriola, ${ }^{3}$ \\ Alan Talevi, ${ }^{1}$ and Carolina Carrillo ${ }^{2}$ \\ ${ }^{1}$ Medicinal Chemistry, Department of Biological Sciences, Faculty of Exact Sciences, National University of La Plata, 47 y 115, \\ La Plata (B1900AJI) Buenos Aires, Argentina \\ ${ }^{2}$ Instituto de Ciencia y Tecnología Dr. César Milstein (ICT Milstein), Argentinean National Council of Scientific and Technical Research \\ (CONICET), Saladillo 2468, Ciudad Autónoma de Buenos Aires (C1440FFX), Argentina \\ ${ }^{3}$ Instituto de Investigaciones Bioquímicas de Buenos Aires, Argentinean National Council of Scientific and Technical Research \\ (CONICET), Avenida Patricias Argentinas 435, Ciudad Autónoma de Buenos Aires (C1405BWE), Argentina
}

Correspondence should be addressed to Alan Talevi; atalevi@biol.unlp.edu.ar

Received 30 August 2013; Accepted 13 November 2013; Published 30 January 2014

Academic Editors: C. Mackereth, A. Paiardini, and X. Qiu

Copyright (C) 2014 Carolina L. Bellera et al. This is an open access article distributed under the Creative Commons Attribution License, which permits unrestricted use, distribution, and reproduction in any medium, provided the original work is properly cited.

Cruzipain $(\mathrm{Cz})$ is the major cysteine protease of the protozoan Trypanosoma cruzi, etiological agent of Chagas disease. A conformation-independent classifier capable of identifying $\mathrm{Cz}$ inhibitors was derived from a 163-compound dataset and later applied in a virtual screening campaign on the DrugBank database, which compiles FDA-approved and investigational drugs. 54 approved drugs were selected as candidates, 3 of which were acquired and tested on $\mathrm{Cz}$ and $\mathrm{T}$. cruzi epimastigotes proliferation. Among them, levothyroxine, traditionally used in hormone replacement therapy in patients with hypothyroidism, showed dosedependent inhibition of $\mathrm{Cz}$ and antiproliferative activity on the parasite.

\section{Introduction}

Chagas disease is a tropical parasitic disease caused by the flagellate protozoan Trypanosoma cruzi. T. cruzi lifecycle includes both vertebrate and invertebrate hosts. 80 to $90 \%$ of infections in humans occur when haematophagous triatomine bug feces come into contact with wounded skin or mucosae [1]. Other infection ways include blood-transfusion and congenital transmission. Even though a series of control campaigns developed by World Health Organization (WHO), Pan American Health Organization (PAHO), and national authorities have considerably reduced Chagas disease incidence in the last fifteen years, there are still almost 8 million infected people and 28 million people at risk [2-4].

Current treatment against Chagas relies on only two drugs developed during 1960s-1970s, namely, nifurtimox and benznidazole, which are not effective in the late chronic phase of the disease and present severe side effects and resistance issues [5-7]. It is worth noting, however, that important advances have been made in the fields of biochemistry and molecular biology of T. cruzi and novel antichagasic therapeutics [4, 8-11]. Cysteine protease inhibitors are among the most investigated candidates against T. cruzi [11]. Cruzipain $(\mathrm{Cz})$, the major cysteine protease of the parasite, has been particularly explored as new drug target (a model of the enzyme is presented in Figure 1). This enzyme has proven to be essential for replication of the intracellular form of T. cruzi and plays a role in host-parasite interactions [12]. It is believed that $\mathrm{Cz}$ inhibition produces accumulation of the inactive precursor of the proteinase within the Golgi complex, which eventually leads to osmotic shock and cell death [13].

Virtual screening encompasses the application of a diversity of computational methods (models or algorithms) to chemical libraries or databases, in order to prioritize which 


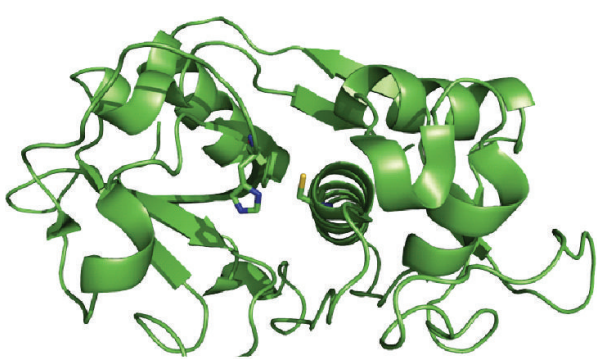

(a)

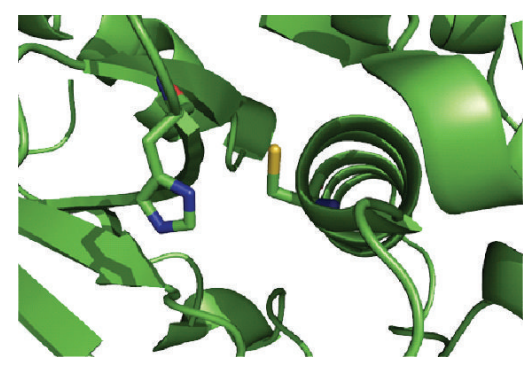

(b)

FIgURE 1: (a) Model of the cruzipain. (b) Active site of cruzipain.

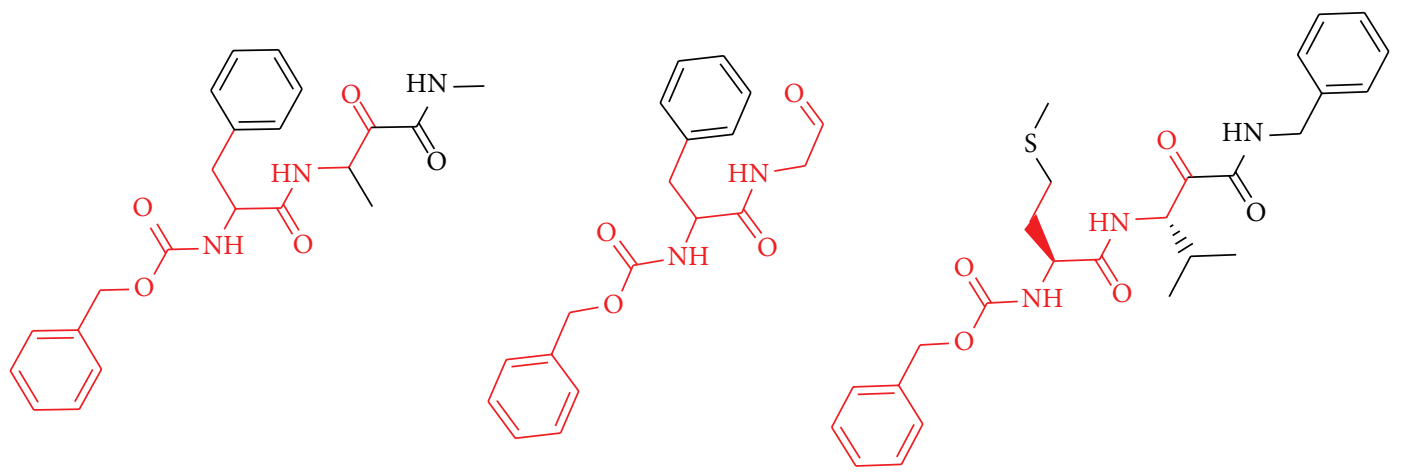

FIgURE 2: An example of MCS. The MCS of the three structures is depicted in red.

of the library compounds will be sent to experimental (in vitro or in vivo) testing. Here, we present a $2 \mathrm{D}$ classification model derived from a 163-compound dataset of $\mathrm{Cz}$ inhibitors and noninhibitors. The model was later applied in a virtual screening (VS) campaign to explore the small molecule database DrugBank in order to identify novel $\mathrm{Cz}$ reversible inhibitors. DrugBank compiles FDA-approved and experimental drugs $[14,15]$, being particularly helpful to conduct VS campaigns aimed to drug repurposing (i.e., searching new therapeutic indications for already known drugs). Traditionally, second medical uses emerged from intelligent exploitation of approved or investigational drugs side effects (e.g., to exploit the aspirin antiplatelet effect to prevent heart attacks and strokes or the use of sildenafil to treat erectile dysfunction). Lately, however, knowledgebased, rational drug repositioning (chemoinformatics- and bioinformatics-based and others) has gained attention [1619] and is being increasingly used to aid in discovering novel treatments for rare, neglected, and poverty-related conditions [20-22].

\section{Materials and Methods}

2.1. Dataset Compilation and Splitting. Building a computational model capable of discriminating active from inactive chemical compounds involves three fundamental steps. First, a dataset of chemical compounds whose class (active or inactive) has been experimentally determined should be compiled. Second, the dataset must be partitioned into a training set that will be used to infer (or train or calibrate) the model and an independent (hold-out) test set that will be used to assess the model predictive ability. Ideally, the training set should present an adequate balance of the active and inactive classes so that the inferred model is not biased. In our case, a 163-compound balanced dataset including $82 \mathrm{Cz}$ reversible inhibitors and 81 noninhibitors was compiled from literature [23-34]. The dataset is available as Supplementary Material available online at http://dx.doi.org/10.1155/2014/279618. A relevant issue that should be addressed is how to split the dataset into representative training and test sets. It has been demonstrated that random partition is an adequate approach whenever training and test sets of similar size are selected, but more rational sampling approaches provide better results when test sets are small compared to the correspondent training sets $[35,36]$. Following the latter criteria, the LibraryMCS v0.7 (ChemAxon) hierarchical clustering approach was applied in combination with the $k$-means clustering implemented in Statistica 10 Cluster Analysis module (Statsoft Inc, 2011). The fundamental idea is to identify, within the diverse dataset, groups of common chemical features to guide the selection of adequate training and test sets. LibraryMCS relies on similarity-guided maximum common substructure (MCS) to cluster a set of chemical structures without exhaustive pairwise comparison. Covalently bonded atoms are regarded as a mathematical "graph" where an atom and a bond correspond to a vertex and an edge, respectively. A common substructure 
is defined as a substructure present in two molecules with the same atom types and bond connections. The MCS is defined as the common substructure with the largest number of atoms or bonds (see Figure 2, e.g.) [37]. Library MCS builds the similarity matrix for the input structures and finds the MCS for the two most similar molecules. This MCS is then used to create and populate a cluster through substructure search. Such procedure is repeated iteratively until no pair of structures with similarity above a similarity threshold is found, in which case singletons are generated. Similarityguided MCS search is used to find MCS of multiple structures efficiently, without exhaustive pairwise comparison. Thus, LibraryMCS leads to reproducible but approximate solutions [38]. Since the number of clusters in $k$-means clustering analysis is a user-defined parameter, hierarchical clustering has been applied here to define an initial partition of " $n$ " objects into " $g$ " groups, as suggested by Everitt et al. [39], and the groups of compounds were later optimized by $k$ means algorithm, minimizing the Euclidean distance to the group centers. A smallest common substructure of at least 9 atoms was used, and a randomly selected member from the clusters defined by the hierarchical approach was used as seed in the k-means clustering procedure. A series of descriptors computed with Dragon 6.0 (Milano Chemometrics, 2012) representing different aspects of molecular structure (viz., molecular weight, $\log P$, polar surface area, number of $\mathrm{H}$ bonds acceptors, information index of atomic content, sum of atomic van der Waals volumes) were normalized and applied to calculate Euclidean distance. Once the clusters were separately identified in the inhibitors and noninhibitors categories, $25 \%$ of each cluster was assigned to an independent test set for validation purposes, while the remaining $75 \%$ of the clusters were retained as training set for modeling purposes. The structures of both training and test set compounds are provided as Supplementary Information.

2.2. Descriptor Calculation and Modeling. Molecular descriptors are numerical variables that reflect different aspects of molecular structure; as such, they are useful to derive quantitative structure-activity relationships. A total of 3764 0D-2D molecular descriptors were computed with Dragon 6.0 Academic version (Milano Chemometrics. 2010). Since the values of such descriptors are conformation independent, they are particularly suitable for their application in VS campaigns, requiring no preprocessing (e.g., conformational analysis or optimization) of the screened database structures. From the 3764 descriptors, 25 random subsets of no more than 254 descriptors were generated, and these subsets were used as descriptor pools for modeling purposes (random subset approach).

Linear Discriminant Analysis (LDA) was then conducted in order to derive a classification model capable of distinguishing $\mathrm{Cz}$ inhibitors from noninhibitors. LDA is a qualitative supervised learning method aimed to finding a linear combination of independent variables to differentiate between two or more categories of objects. Each object class is associated with a given value (an integer value) of an arbitrary variable that serves as class label. In our case, only two object classes (ACTIVE-Cz inhibitors and INACTIVEnoninhibitors) were considered; thus the class label assumes two observed values ( 1 and -1 , resp.). Since the output of the function being searched is not a continuous variable but only an object category, LDA and other classificatory techniques may be useful to handle noisy data, for example, if a given experimental endpoint is associated with large variability or if experimental data from a diversity of laboratories are compiled [40].

The Discriminant Analysis module of Statistica 10 was used to build the models. A tolerance value of 0.5 was selected in order to exclude highly correlated descriptors from the model. All the coefficients linked to the models descriptors were significant at a 0.05 level. A minimum ratio of 15 between the number of training set compounds and the number of independent variables was used in order to reduce the chances of overfitting. Overfitting refers to gaining explanatory power on the training set compounds at the cost of losing predictive ability. Parsimony principle, Wilks' lambda, and the performance of the model on the independent test set were used to select the best model. Standard validation approaches (stratified leave-group out cross-validation, randomization test, and external validation) were used to assess the model's robustness and predictive ability [41]. Stratified 20-fold cross-validation and 30 randomization tests were applied. Cross-validation implies removing training set compounds, regenerating the model and using this new model to predict the removed cases. Randomization implies scrambling the dependent variable among the training set compounds (thus abolishing the structure-property relationship) and obtaining a new, "randomized" model, which should theoretically perform poorly compared to the actual model.

2.3. Simulated Virtual Screening Campaign. An issue that emerges from using a reduced dataset (such as our 42compound test set) to assess the performance of ranking methods in virtual screening is that the metrics used to such purpose exhibit a higher variance compared to significantly large datasets. Experiments conducted by Truchon and Bayly [42] show that the standard deviations associated with several frequently used metrics (among them the ROCAUC) are higher for small datasets and converge to a constant value when the size of the dataset increases.

Another problem is related to the high ratio of inactives which mainly hinders the early recognition ability in what is known as the "saturation effect." That is, for datasets with a high ratio of hits (in our case, $\mathrm{Cz}$ inhibitors), once hit compounds saturate the early part of the ordered list, the enrichment metric cannot get any higher. To estimate in a more realistic way the utility of our model in a real virtual screening approach, we have dispersed our test set among 444 putative noninhibitors acting as decoys. Such putative noninhibitors are highly similar compounds $(0.95$ similarity or more) to the test set noninhibitors and have been retrieved from PubChem. This simulated database thus contains 21 known Cz-inhibitors among 465 known or putative noninhibitors; that is, the $\mathrm{Cz}$-inhibitors ratio is 
less than 0.05 , representing a more challenging set to assess the enrichment ability of our models. Note that some of these putative noninhibitors (decoys) might actually be $\mathrm{Cz}$ inhibitors; thus, the true performance of our models may be even higher than the one obtained through this simulated experiment.

2.4. Virtual Screening. DrugBank 3.0, a chemical database which compiles FDA approved and investigational drugs, was screened. Only approved and experimental small molecules and nutraceuticals (6684 total compounds) were considered (biotech drugs were excluded a priori). Pharmacological Distribution Diagrams (PDD) and separate Receiver Operating Characteristic (ROC) curves were constructed for both the training and test sets, in order to select the discrimant function score threshold value determining and adequate sensitivity/specificity ration, to be used in the VS campaign $[43,44]$. ROC curves provide graphical insight into the specific-sensitivity balance for different model score thresholds, allowing selection of an appropriate threshold on the basis of context-dependent criteria. To build ROC curves MedCalc ROC curves analysis tool was applied (MedCalc software, 2012).

2.5. Inhibitory Effect on Cz Activity Assay. To study the effect of the selected compounds on $\mathrm{Cz}$ activity, the enzyme was partially purified by ammonium sulfate precipitation followed by affinity column chromatography on concanavalin A-sepharose (Sigma), as previously described [45]. The activity of the partially purified $\mathrm{Cz}$ was assayed with $250 \mu \mathrm{M}$ Bz-Pro-Phe-Arg-pNA (Sigma) as substrate, incubated in a buffer of $6,5 \mu \mathrm{M}$ dithiothreitol (DTT) and $50 \mathrm{mM}$ Tris- $\mathrm{HCl}$ pH 7 [46], in presence or absence of diverse compounds. The reaction was measured spectrophotometrically at room temperature at $410 \mathrm{~nm}$ for $5 \mathrm{~min}$ (Beckman CoulterTM DU530 Life Science UV-vis spectrophotometer). The values obtained were converted into pmol of hydrolyzed substrate per min by using the extinction coefficient $8.800 \mathrm{M}^{-1} \mathrm{~cm}^{-1}$ (p-nitroanilines). The inhibitory effect of the selected candidates was expressed as a percentage of residual activity of $\mathrm{Cz}$ with respect to the assay without inhibitors.

2.6. Inhibitory Effects on T. cruzi Epimastigote Proliferation. Epimastigotes of the T. cruzi strain Y were cultured at $28^{\circ} \mathrm{C}$ in BHT medium with $20 \mathrm{mg} / \mathrm{L}$ Haemin, $20 \%$ heat-inactivated fetal calf serum and antibiotics $(100 \mu \mathrm{g} / \mathrm{mL}$ streptomycin and $100 \mathrm{U} / \mathrm{mL}$ penicillin) [47] adding the indicated levothyroxine concentration $(0-200 \mu \mathrm{M})$. Cultures were initiated at $10^{6}$ cells $/ \mathrm{mL}$, and the proliferation was followed daily by cell counting in a hemocytometer chamber. In order to follow the effect of the drug on proliferation for long periods, once the cultures reached the stationary phase (each 7 days), they were restarted by dilution at $10^{7}$ cells $/ \mathrm{mL}$ with fresh medium plus levothyroxine.

\section{Results and Discussion}

Clustering procedure revealed 5 groups of at least $6 \mathrm{com}$ pounds in the ACTIVE category and 7 groups of at least 7 compounds in the INACTIVE class. According to MCS clustering, there are 4 compounds in the ACTIVE class which can be considered outliers (singletons or groups of only two compounds, meaning they share no MCS above the specified number of atoms with other molecules from the set), whereas the INACTIVE category presents 14 outliers. On the basis of the clustering procedure, $25 \%$ of each cluster was assigned to the test set for external validation purposes, while the remaining $75 \%$ of each cluster was assigned to the training set upon which the model was derived.

The following model was obtained through LDA:

$$
\begin{aligned}
& \text { Class }= 0.819+0.536 * \mathrm{VE} 1 \_\mathrm{X}-1.187 * \mathrm{C}-018 \\
&+0.495 * \mathrm{~F}-048-0.688 * \mathrm{Nsss} \mathrm{N}-0.187 * \mathrm{H}-051 \\
&-0.427 * \mathrm{O}-056-0.09 * \mathrm{Sds}-5.311 * \text { SpPosA_A }, \\
&(N=121, \lambda=0.445, F(8,112)=17.476, p<0.00001),
\end{aligned}
$$

where VE1 X represents the sum of the coefficients of the eigenvector associated with the last (largest negative) eigenvalue of the chi matrix. Such matrix is a modified adjacency matrix, obtained by weighting each bond between pairs of vertices by the edge connectivity [48]. The elements of the chi matrix are define as follows:

$$
X_{i, j}= \begin{cases}\frac{1}{\sqrt{m \times n}} & \text { if } i, j \text { are adjacent } \\ 0 & \text { otherwise }\end{cases}
$$

where $m$ and $n$ are the valences of the vertices involved. $\mathrm{C}-018$ is the number of $=\mathrm{CHX}$ (= representing a double bound and $\mathrm{X}$ being a heteroatom), F-084 denotes the number of atoms $\mathrm{F}$ attached to carbons SP2, NsssN represents the number of tertiary nitrogens, $\mathrm{H}-051$ is the number of $\mathrm{H}$ atoms attached to an alpha carbon, O-056 denotes the number of groups $\mathrm{OH}$, Sds symbolizes the sum of the E-states of the $=S$ atom type (thus providing information about the electron accessibility and the count of the number of atoms of such atom-type) and SpPosA A is the normalized spectral positive sum from adjacency matrix. The magnitudes of the beta coefficients of such descriptors are, respectively, 0.564, $0.185,0.168,0.160,0.137,0.135,0.142$, and 0.108 , showing that VE1_X is the most relevant independent variable of the model. It should be highlighted that the model presents a good cases per predictor ratio (around 15) which indicates a low chance of overfitting, as confirmed later in the external validation results. When using 0 as a score threshold to differentiate active from inactive compounds, the model presents $87 \%$ of good classifications among the training set inactive compounds, $90 \%$ of good classifications among the training set active compounds, and an overall of $88 \%$ good classifications. Regarding the test set, the model accurately classifies $81 \%$ of the active and $90.5 \%$ of the inactive compounds, with an overall good classification of $86 \%$. These 
results seem to confirm that no overfitting has occurred, since the performance on the test set is very similar to the performance on the training set. The average performance of the randomized models was $68.4(\mathrm{sd}=4.1)$ showing that the randomized models were significantly outperformed by the actual model, as expected. Cross-validation resulted in an average percentage of good-classifications of 79\% (average of the result of the 20 folds); remarkably, among the worstclassified folds we found 5 of the outliers detected by the hierarchical clustering procedure.

We resorted to PDD and ROC curves in order to optimize the chosen threshold score on a rational basis [40, 41]. Figures 3 and 4 present, respectively, the PDDs of the training and test sets, the ROC curves for the training set, test set, and the 486-compound simulated database. The area under the curve (AUC) for the training and test sets ROC curves was, respectively, 0.930 and 0.923 ( 1 represents perfect classification, while 0.5 represents random classification). 0.06 was selected as the cutoff value to differentiate active from inactive compounds in the VS campaign. According to the ROC curves data, this value corresponds to a sensitivity of $87 \%$ and a specificity of $88 \%$ in the training set, and a sensitivity of $81 \%$ and a specificity of $95 \%$ in the test set. As stated by Triballeau in the original application of ROC curves to VS [41], the selection of a given balance between sensitivity and specificity is not a statistical matter but a context-dependent decision. In our case, due to a limited budget to acquire and test compounds, we have prioritized specificity (i.e., reducing the chance of false positives) over sensitivity. This means that, in order to increase the chance of having positive results in the biological tests, we risk losing potentially valuable structural motifs.

Remarkably, our simulated VS campaign resulted in a ROC AUC of 0.953 (larger, in fact, that the ROC AUCs obtained for the training and test sets). Furthermore, 16 out of $21 \mathrm{Cz}$ inhibitors in the dataset $(76 \%$ of the total number of inhibitors) appear among the $5 \%$ best ranked compounds from the simulated database.

From 6684 small approved and investigational molecules of the DrugBank 3.0 database, 64 candidates were selected, with a score above the selected threshold; 54 of them correspond to approved drugs, which are the straightforward candidates for repositioning purposes. On the basis of their accessibility, 3 of them (Figure 5) were acquired and experimentally tested in the enzymatic assay on Cz. The acquired candidates were cisapride (gastroprokinetic agent, increases motility in the gastrointestinal tract), paroxetine (antidepressant, a selective inhibitor of serotonin reuptake), and levothyroxine (used in hormone replacement therapy in patients with hypothyroidism). Among them, only paroxetine had been previously tested on cruzipain (with negative results) through a quantitative high-throughput screening approach which assessed the effect of more than 197,000 candidates on the hydrolysis of the fluorogenic substrate ZPhe-Arg-AMC [49].

Using Bz-Pro-Phe-Arg-pNA as chromogenic substrate, levothyroxine showed a significant inhibitory effect on $T$. cruzi $\mathrm{Cz}$ activity (Figure 6(a)). Such inhibition proved to be

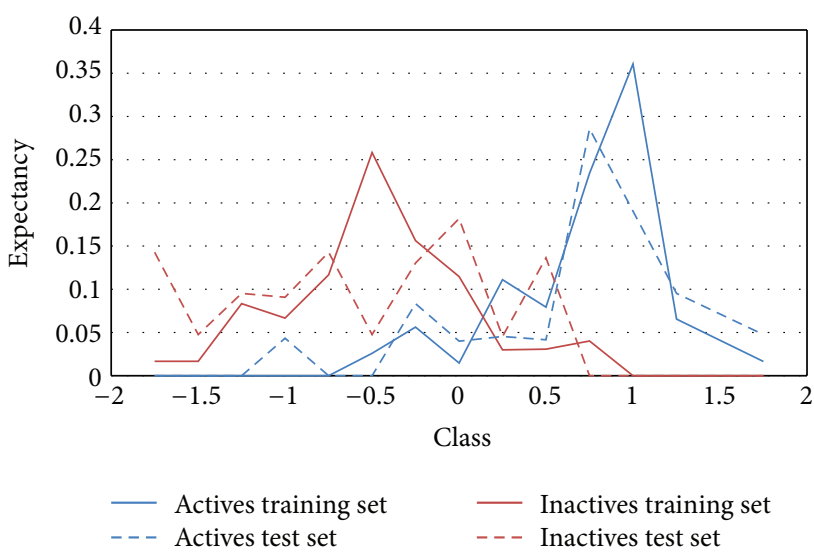

FIgURE 3: PDD showing the distribution of training and test set active and inactive compounds along the score values of the model. An acceptable superposition between both sets can be observed.

dose dependent on purified $\mathrm{Cz}$, with an IC50 of $38.43 \pm$ $6.82 \mu \mathrm{M}$ (Figure 6(b)).

T. cruzi epimastigotes proliferation was affected by levothyroxine progressively in time and in a dose-dependent manner (Figure 7). The effect was clearly notorious on the third week of assay showing a median inhibitory dose $\left(\mathrm{ID}_{50}\right)$ of $121.76 \pm 11.39 \mu \mathrm{M}$ at the middle log phase of controls (17th day).

\section{Conclusions}

An 8-descriptor conformation-independent classification model was derived from a 163-compound dataset which compiled $\mathrm{Cz}$ inhibitors and noninhibitors extracted from the literature. The model presented an excellent case to descriptor ratio and similar performance on both the training and the test sets which suggest good predictive ability and absence of overfitting. Since only conformation-independent descriptors were included in the model, it is particularly suitable for efficient exploration of drug libraries through VS campaigns without requiring any preprocessing of the library structures.

Having in mind the potential of knowledge-based drug repositioning to develop novel therapies for neglected and rare diseases, the model was applied in a VS campaign to select potential antichagasic drugs from the DrugBank database, which compiles approved and investigational active ingredients. PDD and ROC curve analysis were conducted in order to select a score cutoff value to differentiate active and inactive agents on a rational basis.

Three candidates were acquired and experimentally tested in enzymatic and inhibitory assays. Among them, levothyroxine (traditionally used in hormone replacement therapy in patients with hypothyroidism) showed a dosedependent inhibition on $\mathrm{Cz}$ activity with concomitant effects on T. cruzi proliferation. The results exemplify the potential of computer-aided drug repositioning in the search of novel medications for poverty-related diseases. 


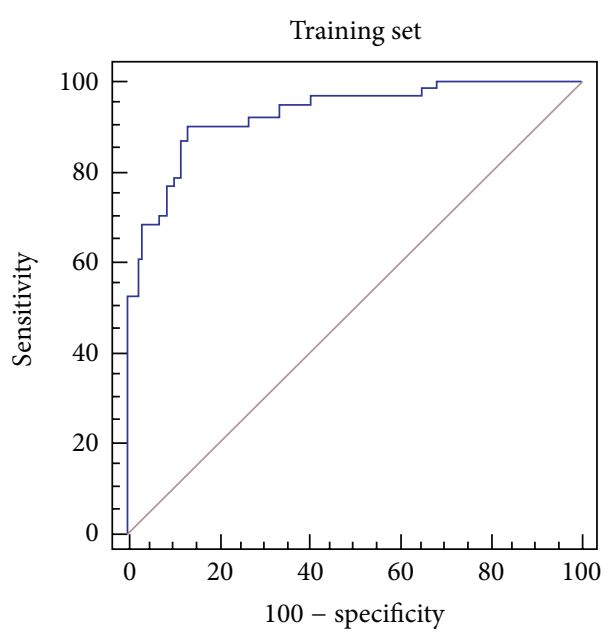

(a)

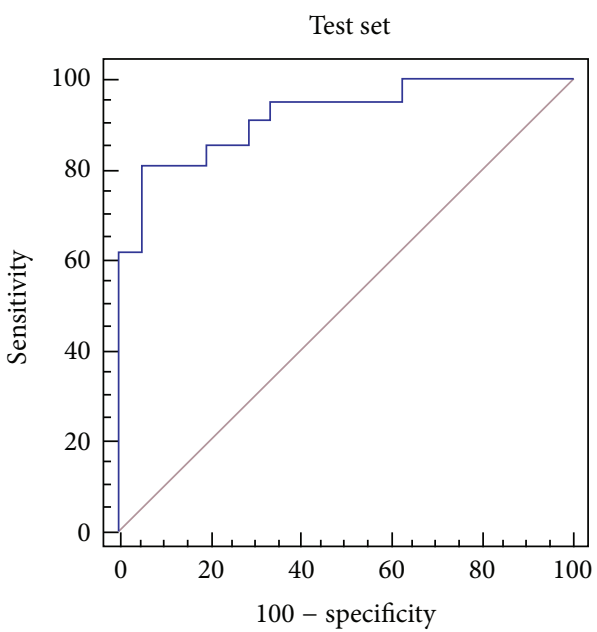

(b)

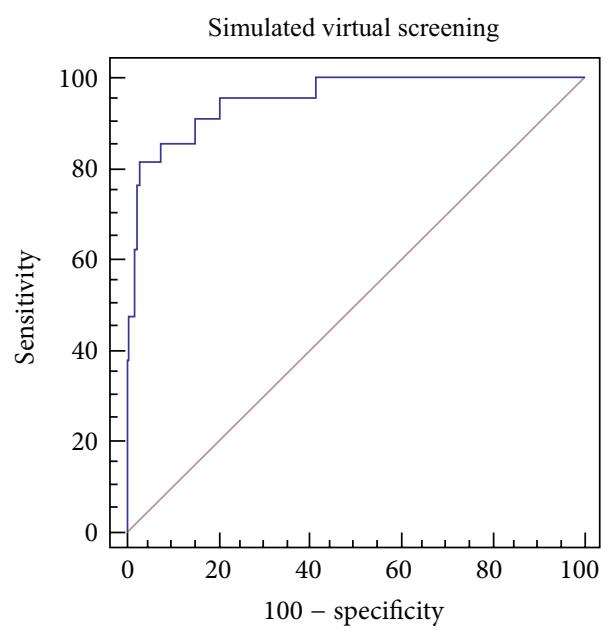

(c)

Figure 4: Training set (a), test set (b), and simulated database (c) ROC curves.<smiles>COc1cc(N)c(Cl)cc1C(=O)NC1CCN(CCCOc2ccc(F)cc2)CC1OC</smiles><smiles>Fc1ccc(C2CCNCC2COc2ccc3c(c2)OCO3)cc1</smiles><smiles>N[C@@H](Cc1cc(I)c(Oc2cc(I)c(O)c(I)c2)c(I)c1)C(=O)O</smiles>

Levothyroxine

FIgURE 5: Molecular structures of the three candidates selected for enzymatic testing. 


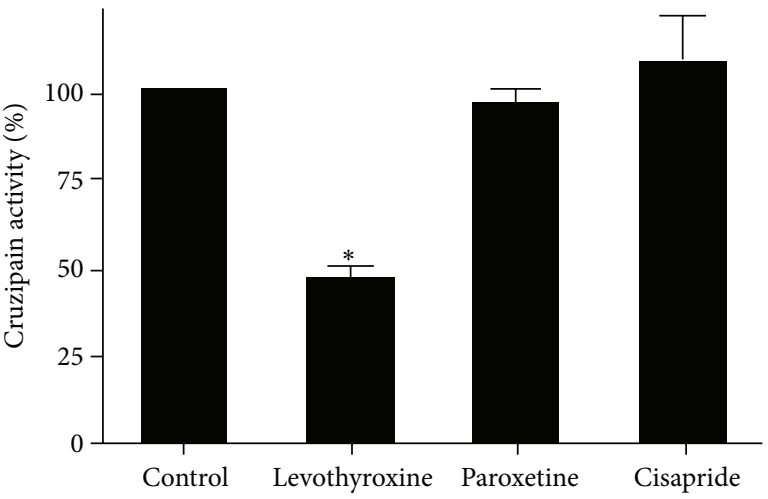

(a)

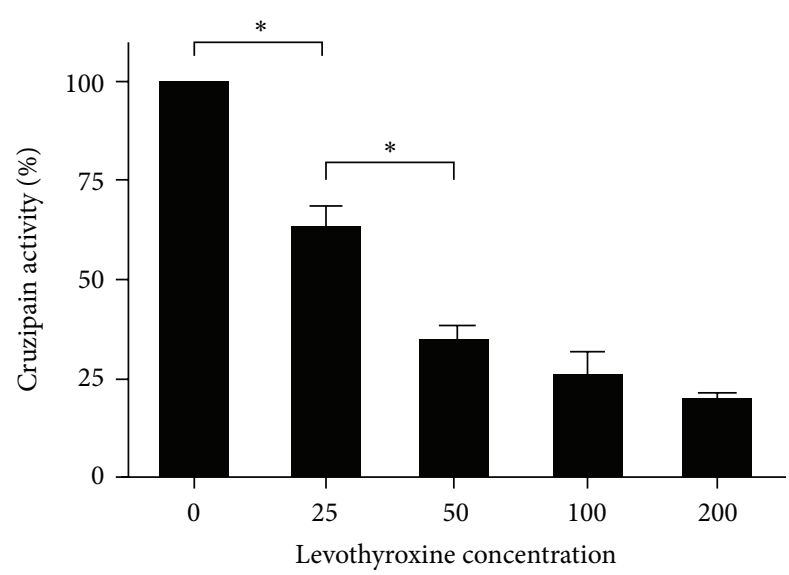

(b)

FIGURE 6: (a) Inhibitory effect of the three selected candidates on purified $\mathrm{Cz}$ activity. The final concentration of each compound was $50 \mu \mathrm{M}$. Protease activity is expressed as percentage of the control condition (2\% DMSO). (b) Dose-dependent inhibitory effect of levothyroxine on purified $\mathrm{Cz}$ activity. Levothyroxine was assayed in a concentration range of $0-200 \mu \mathrm{M}$. Remanent $\mathrm{Cz}$ activity was expressed as a percentage respect to the control $(0 \mu \mathrm{M}$ levothyroxine, $2 \% \mathrm{DMSO})$. Asterisks indicate significant differences $\left({ }^{*} P<0.05\right)$.

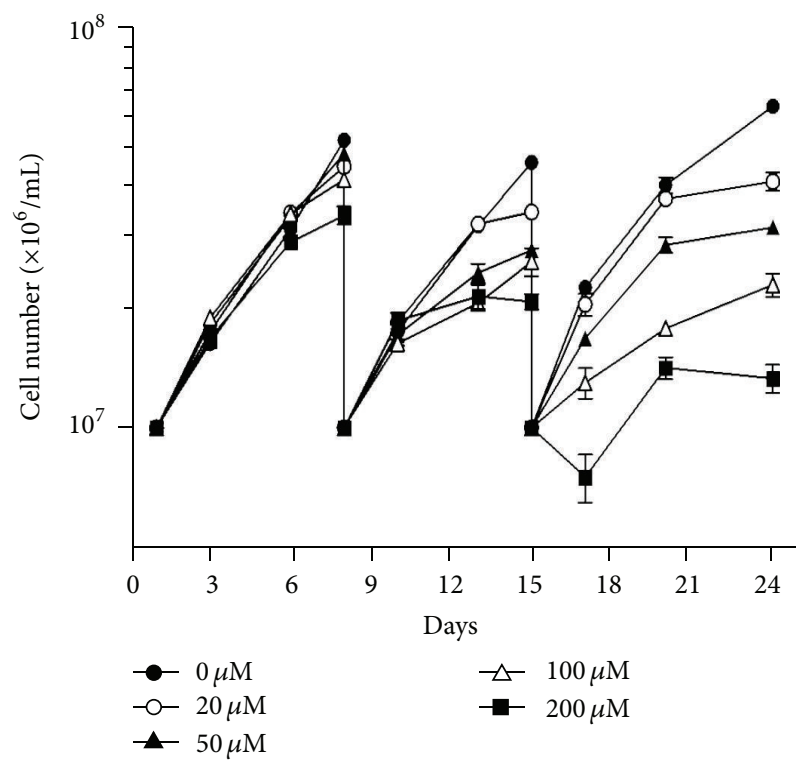

FIGURE 7: Effect of levothyroxine on T. cruzi epimastigotes proliferation. To determine the growth rate, $10^{7}$ cells $/ \mathrm{mL}$ were seeded in BHT medium and cultured at $28^{\circ} \mathrm{C}$ with the indicated concentrations of the drug; the control condition was done with $0 \mu \mathrm{M}$ levothyroxine, 2\% DMSO. Parasites were counted daily using a hemocytometer chamber and, once they reached the stationary phase, were rediluted at $10^{7}$ cells $/ \mathrm{mL}$ with fresh medium plus the indicated levothyroxine concentration. Results represent the mean \pm SD of a representative experiment.

\section{Abbreviations}

LDA: Linear discriminant analysis

MCS: Maximum common substructure

ROC: Receiving operating characteristic

VS: Virtual screening.

\section{Conflict of Interests}

The authors declare no conflict of interests related to the content of this paper.

\section{Authors' Contribution}

The paper was written through contributions of all authors. All authors have given approval to the final version of the paper.

\section{Acknowledgments}

Carolina L. Bellera and Darío E. Balcazar are fellowship holders from the National Council of Scientific and Technical Research (CONICET). Carolina Carrillo, Carlos A. Labriola, and Alan Talevi are members of CONICET. This work was supported by the Faculty of Exact Sciences of the National University of la Plata, the National University of La Plata (UNLP), the Commission of Scientific Research of Buenos Aires Province (CIC) (PIRPS Projects and Incentivos UNLP), UBACyT-GF 20020100200029, University of Buenos Airesg, PIP 20100685 from the National Council of Scientific and Technical Research (CONICET), and IANAS Fellowship Seed Grant 2013.

\section{References}

[1] J. R. Coura and S. L. De Castro, "A critical review on chagas disease chemotherapy," Memorias do Instituto Oswaldo Cruz, vol. 97, no. 1, pp. 3-24, 2002.

[2] Á. Moncayo and A. C. Silveira, "Current epidemiological trends for Chagas disease in Latin America and future challenges in epidemiology, surveillance and health policy," Memorias do Instituto Oswaldo Cruz, vol. 104, supplement 1, pp. 17-30, 2009. 
[3] World Health Organization, Control of Chagas disease. Second report of the WHO Expert Committee, 2002.

[4] TDR, "Special Programme for Research and Training in tropical diseases), World Health Organiza-tion," Research priorities for Chagas disease, human African trypanosomiasis and leishmaniasis, 2012.

[5] J. A. Urbina and R. Docampo, "Specific chemotherapy of Chagas disease: controversies and advances," Trends in Parasitology, vol. 19, no. 11, pp. 495-501, 2003.

[6] L. Murcia, B. Carrilero, and M. Segovia, "Limitations of currently available Chagas disease chemotherapy," Revista Española De Quimioterapia, vol. 25, no. 1, pp. 1-3, 2012.

[7] F. Astelbauer and J. Walochnik, "Antiprotozoal compounds: state of the art and new developments," International Journal of Antimicrobial Agents, vol. 38, no. 2, pp. 118-124, 2011.

[8] F. Villalta, J. Scharfstein, A. W. Ashton et al., "Perspectives on the Trypanosoma cruzi-host cell receptor interactions," Parasitology Research, vol. 104, no. 6, pp. 1251-1260, 2009.

[9] P. Cuervo, G. B. Domont, and J. B. De Jesus, "Proteomics of trypanosomatids of human medical importance," Journal of Proteomics, vol. 73, no. 5, pp. 845-867, 2010.

[10] F. Sánchez-Sancho, N. E. Campillo, and J. A. Páez, "Chagas disease: progress and new perspectives," Current Medicinal Chemistry, vol. 17, no. 5, pp. 423-452, 2010.

[11] V. G. Duschak and A. S. Couto, "An insight on targets and patented drugs for chemotherapy of chagas disease," Recent Patents on Anti-Infective Drug Discovery, vol. 2, no. 1, pp. 19-51, 2007.

[12] V. G. Duschak and A. S. Couto, "Cruzipain, the major cysteine protease of Trypanosoma cruzi: a sulfated glycoprotein antigen as relevant candidate for vaccine development and drug target. A review," Current Medicinal Chemistry, vol. 16, no. 24, pp. 31743202, 2009.

[13] G. C. Rodrigues, A. P. Aguiar, J. L. da Silva Gonçalves Vianez Jr., A. Macrae, A. C. N. de Melo, and A. B. Vermelho, "Peptidase inhibitors as a possible therapeutic strategy for chagas disease," Current Enzyme Inhibition, vol. 6, no. 4, pp. 183-194, 2010.

[14] C. Knox, V. Law, T. Jewison et al., "DrugBank 3.0: a comprehensive resource for 'Omics' research on drugs,' Nucleic Acids Research, vol. 39, no. 1, pp. D1035-D1041, 2011.

[15] D. S. Wishart, C. Knox, A. C. Guo et al., "DrugBank: a comprehensive resource for in silico drug discovery and exploration," Nucleic Acids Research, vol. 34, pp. D668-672, 2006.

[16] F. Gan, B. Cao, D. Wu, Z. Chen, T. Hou, and X. Mao, "Exploring old drugs for the treatment of hematological malignancies," Current Medicinal Chemistry, vol. 18, no. 10, pp. 1509-1514, 2011.

[17] A. Talevi, E. A. Castro, and L. E. Bruno-Blanch, "Virtual screening: an emergent, key methodology for drug development in an emergent continent-a bridge towards patentability," in Advances Methods and Applications in Chmoinformatics: Research Progress and New Applications, E. A. Castro and A. K. Haghi, Eds., pp. 229-246, IGI Global, Hershey, Pa, USA, 1st edition, 2011.

[18] S. N. Deftereos, C. Andronis, E. J. Friedla, A. Persidis, and A. Persidis, "Drug repurposing and adverse event prediction using high-throughput literature analysis," Wiley Interdisciplinary Reviews: Systems Biology and Medicine, vol. 3, no. 3, pp. 323334, 2011.

[19] Y. A. Lussier and J. L. Chen, "The emergence of genome-based drug repositioning," Science Translational Medicine, vol. 3, no. 96, Article ID 96ps35, 2011.
[20] S. Ekins, A. J. Williams, M. D. Krasowski, and J. S. Freundlich, "In silico repositioning of approved drugs for rare and neglected diseases," Drug Discovery Today, vol. 16, no. 7-8, pp. 298-310, 2011.

[21] D. Sardana, C. Zhu, M. Zhang, R. C. Gudivada, L. Yang, and A. G. Jegga, "Drug repositioning for orphan diseases," Briefings in Bioinformatics, vol. 12, no. 4, pp. 346-356, 2011.

[22] M. P. Pollastri and R. K. Campbell, "Target repurposing for neglected diseases," Future Medicinal Chemistry, vol. 3, no. 10, pp. 1307-1315, 2011.

[23] J. M. dos Santos Filho, A. C. L. Leite, B. G. D. Oliveira et al., "Design, synthesis and cruzain docking of 3-(4-substitutedaryl)-1,2,4-oxadiazole- $\mathrm{N}$-acylhydrazones as anti-Trypanosoma cruzi agents," Bioorganic and Medicinal Chemistry, vol. 17, no. 18, pp. 6682-6691, 2009.

[24] M. Z. Hernandes, M. M. Rabello, A. C. L. Leite et al., "Studies toward the structural optimization of novel thiazolylhydrazonebased potent antitrypanosomal agents," Bioorganic and Medicinal Chemistry, vol. 18, no. 22, pp. 7826-7835, 2010.

[25] C. R. Rodrigues, T. M. Flaherty, C. Springer, J. H. McKerrow, and F. E. Cohen, "CoMFA and HQSAR of acylhydrazide cruzain inhibitors," Bioorganic and Medicinal Chemistry Letters, vol. 12, no. 11, pp. 1537-1541, 2002.

[26] L. Huang, L. S. Brinen, and J. A. Ellman, "Crystal structures of reversible ketone-Based inhibitors of the cysteine protease cruzain," Bioorganic and Medicinal Chemistry, vol. 11, no. 1, pp. 21-29, 2003.

[27] Y. Choe, L. S. Brinen, M. S. Price et al., "Development of $\alpha$ keto-based inhibitors of cruzain, a cysteine protease implicated in Chagas disease," Bioorganic and Medicinal Chemistry, vol. 13, no. 6, pp. 2141-2156, 2005.

[28] C. Beaulieu, E. Isabel, A. Fortier et al., "Identification of potent and reversible cruzipain inhibitors for the treatment of Chagas disease," Bioorganic and Medicinal Chemistry Letters, vol. 20, no. 24, pp. 7444-7449, 2010.

[29] X. Du, E. Hansell, J. C. Engel, C. R. Caffrey, F. E. Cohen, and J. H. McKerrow, "Aryl ureas represent a new class of antitrypanosomal agents," Chemistry and Biology, vol. 7, no. 9, pp. 733-742, 2000.

[30] K. Brak, P. S. Doyle, J. H. McKerrow, and J. A. Ellman, "Identification of a new class of nonpeptidic inhibitors of cruzain," Journal of the American Chemical Society, vol. 130, no. 20, pp. 6404-6410, 2008.

[31] B. T. Mott, R. S. Ferreira, A. Simeonov et al., "Identification and optimization of inhibitors of trypanosomal cysteine proteases: cruzain, rhodesain, and TbCatB," Journal of Medicinal Chemistry, vol. 53, no. 1, pp. 52-60, 2010.

[32] R. S. Ferreira, A. Simeonov, A. Jadhav et al., "Complementarity between a docking and a high-throughput screen in discovering new cruzain inhibitors," Journal of Medicinal Chemistry, vol. 53, no. 13, pp. 4891-4905, 2010.

[33] K. K. H. Ang, J. Ratnam, J. Gut et al., "Mining a cathepsin inhibitor library for new antiparasitic drug leads," PLoS Neglected Tropical Diseases, vol. 5, no. 5, Article ID e1023, 2011.

[34] K. A. Scheidt, W. R. Roush, J. H. McKerrow, P. M. Selzer, E. Hansell, and P. J. Rosenthal, "Structure-based design, synthesis and evaluation of conformationally constrained cysteine protease inhibitors," Bioorganic and Medicinal Chemistry, vol. 6, no. 12, pp. 2477-2494, 1998.

[35] A. Golbraikh, M. Shen, Z. Xiao, Y.-D. Xiao, K.-H. Lee, and A. Tropsha, "Rational selection of training and test sets for the 
development of validated QSAR models," Journal of ComputerAided Molecular Design, vol. 17, no. 2-4, pp. 241-253, 2003.

[36] A. Golbraikh and A. Tropsha, "Predictive QSAR modeling based on diversity sampling of experimental datasets for the training and test set selection," Molecular Diversity, vol. 5, no. 4, pp. 231-243, 2000.

[37] T. Kawabata, "Build-up algorithm for atomic correspondence between chemical structures," Journal of Chemical Information and Modeling, vol. 51, no. 8, pp. 1775-1782, 2011.

[38] R. Hariharan, A. Janakiraman, R. Nilakantan et al., "MultiMCS: a fast algorithm for the maximum common substructure problem on multiple molecules," Journal of Chemical Information and Modeling, vol. 51, no. 4, pp. 788-806, 2011.

[39] B. S. Everitt, S. Landau, M. Leese, and D. Stahl, "Optimization clustering techniques," in Cluster Analysis, D. J. Balding, N. A. C. Cressie, G. M. Fitzmaurice et al., Eds., pp. 111-142, John Wiley \& Sons, Chichester, UK, 5th edition, 2011.

[40] A. Talevi, C. L. Bellera, M. E. Di Ianni, P. R. Duchowicz, L. E. Bruno-Blanch, and E. A. Castro, "An integrated drug development approach applying topological descriptors," Computers Aid Drug Design and Discovery, vol. 8, pp. 172-181, 2012.

[41] A. Yasri and D. Hartsough, "Toward an optimal procedure for variable selection and QSAR model building," Journal of Chemical Information and Computer Sciences, vol. 41, no. 3-6, pp. 1218-1227, 2001.

[42] J.-F. Truchon and C. I. Bayly, "Evaluating virtual screening methods: good and bad metrics for the "early recognition" problem," Journal of Chemical Information and Modeling, vol. 47, no. 2, pp. 488-508, 2007.

[43] J. Gálvez, R. García-Domenech, C. De Gregorio Alapont, J. V. De Julián-Ortiz, and L. Popa, "Pharmacological distribution diagrams: a tool for de novo drug design," Journal of Molecular Graphics, vol. 14, no. 5, pp. 272-276, 1997.

[44] N. Triballeau, F. Acher, I. Brabet, J.-P. Pin, and H.-O. Bertrand, "Virtual screening workflow development guided by the "receiver operating characteristic" curve approach. Application to high-throughput docking on metabotropic glutamate receptor subtype 4," Journal of Medicinal Chemistry, vol. 48, no. 7, pp. 2534-2547, 2005.

[45] C. Labriola, M. Sousa, and J. J. Cazzulo, "Purification of the major cysteine proteinase (cruzipain) from Trypanosoma cruzi by affinity chromatography," Biological Research, vol. 26, no. 1-2, pp. 101-107, 1993.

[46] J. J. Cazzulo, M. C. Cazzulo Franke, J. Martinez, and B. M. Franke De Cazzulo, "Some kinetic properties of a cysteine proteinase (cruzipain) from Trypanosoma cruzi," Biochimica et Biophysica Acta, vol. 1037, no. 2, pp. 186-191, 1990.

[47] J. J. Cazzulo, B. M. Franke de Cazzulo, J. C. Engel, and J. J. B. Cannata, "End products and enzyme levels of aerobic glucose fermentation in trypanosomatids," Molecular and Biochemical Parasitology, vol. 16, no. 3, pp. 329-343, 1985.

[48] M. Randić, "Similarity based on extended basis descriptors," Journal of Chemical Information and Computer Science, vol. 32, pp. 686-692, 1992.

[49] A. Jadhav, R. S. Ferreira, C. Klumpp et al., "Quantitative analyses of aggregation, autofluorescence, and reactivity artifacts in a screen for inhibitors of a thiol protease," Journal of Medicinal Chemistry, vol. 53, no. 1, pp. 37-51, 2010. 

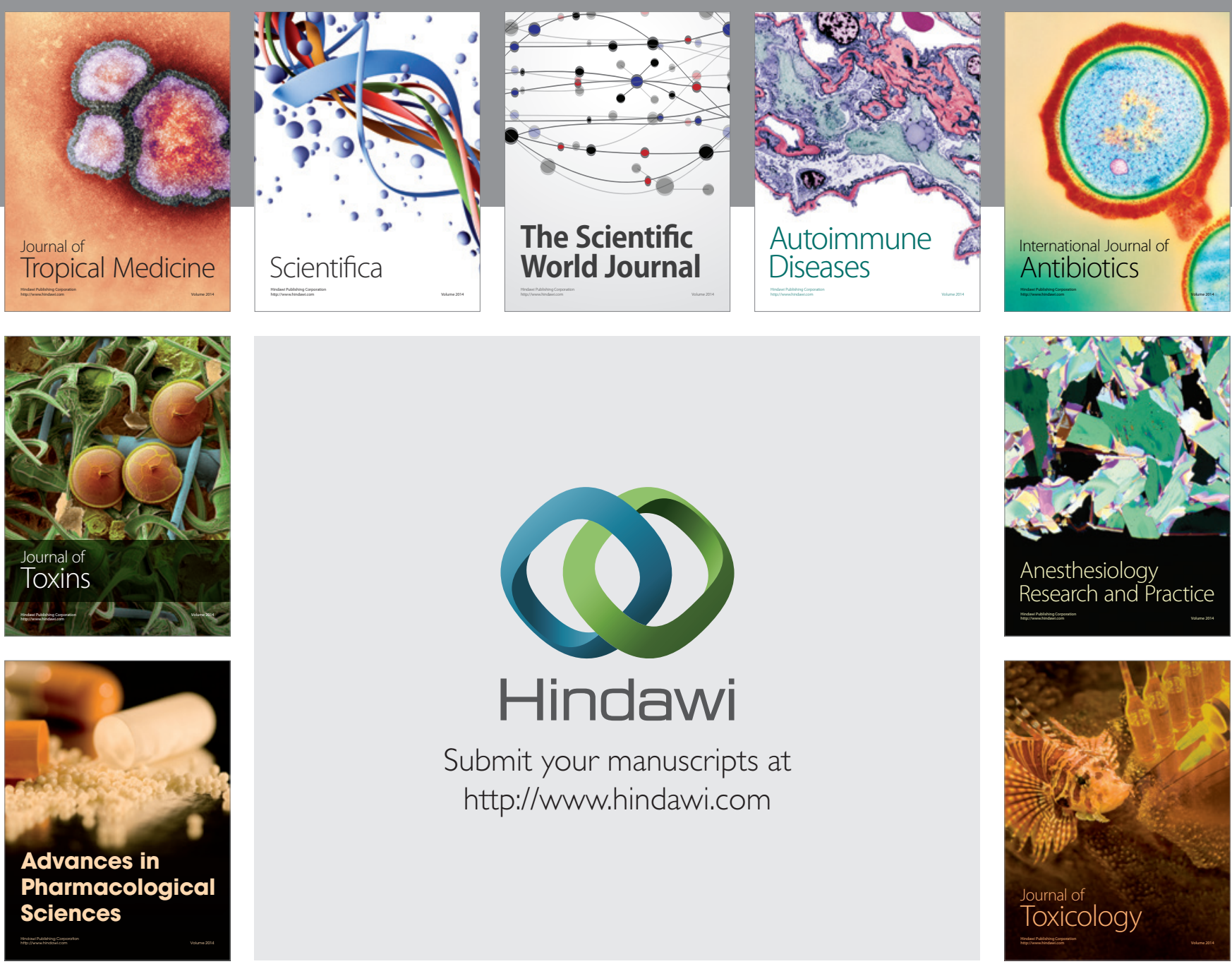

\section{Hindawi}

Submit your manuscripts at

http://www.hindawi.com
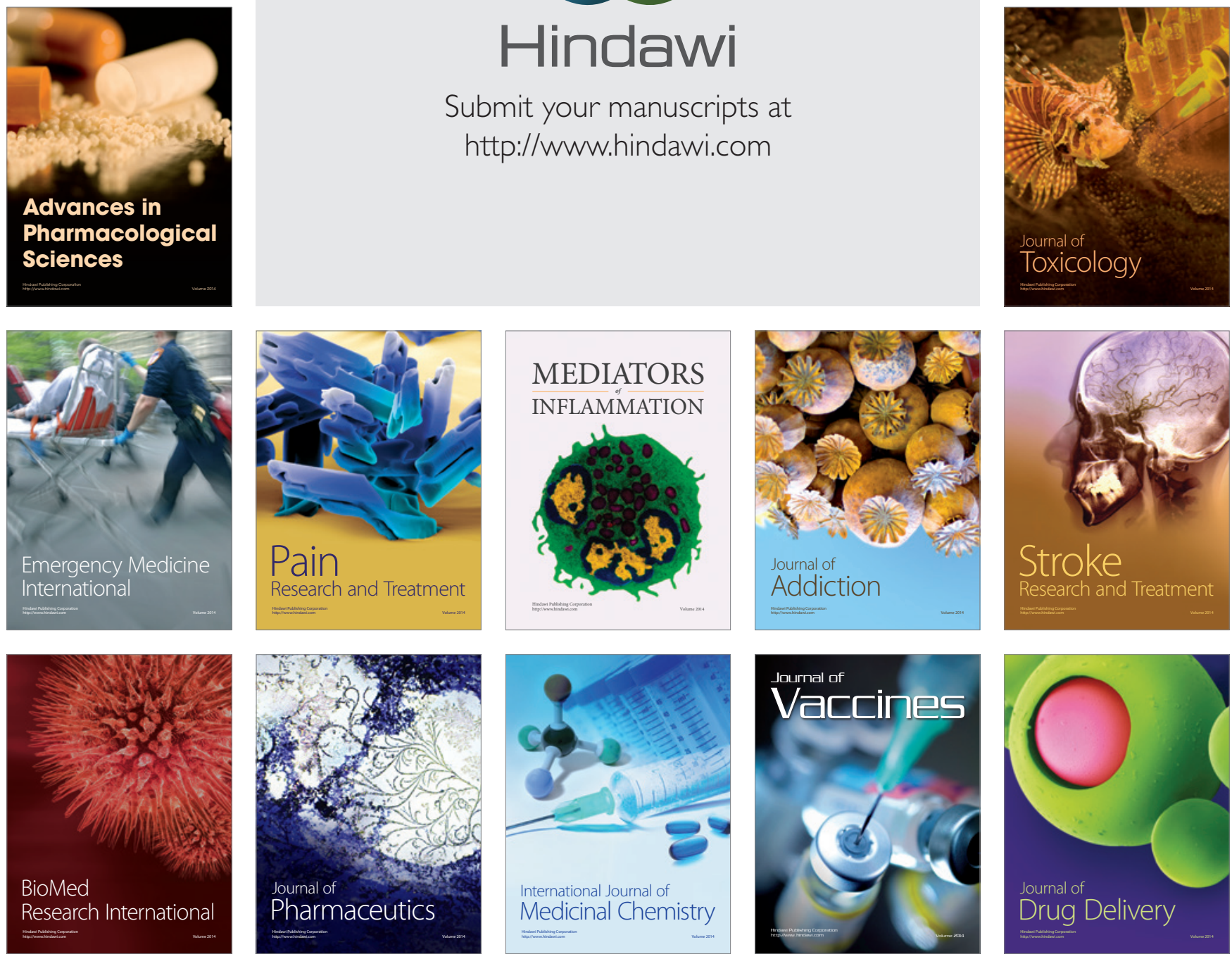